\title{
Diversity, pathogenicity and pandemic potential of Henipavirus: an interview with Benhur Lee
}

\author{
Benhur Lee*,1 \\ ${ }^{1}$ Department of Microbiology, Ward-Coleman Chair in Microbiology, Icahn School of Medicine at Mount Sinai, 1468 Madison Ave, \\ Annenberg Bldg 17-24 New York, NY 10029-6508, USA \\ *Author for correspondence: e.colvin@futuremedicine.com
}

\begin{abstract}
Biography: Dr Benhur Lee is a Professor of Microbiology at the Icahn School of Medicine at Mount Sinai (ISMMS, NY, USA). He obtained his MD from Yale University School of Medicine (1995) and completed his clinical/postdoctoral training at the University of Pennsylvania (1995-2001). He was a Professor in the Department of Microbiology, Immunology \& Molecular Genetics at the David Geffen School of Medicine at UCLA (2001-2013). Dr Lee is an appointed member of the NIH Novel and Exceptional Technology and Research Advisory Committee (NExTRAC), formerly known as the recombinant DNA Advisory committee (RAC). He is also on the International Committee on Taxonomy of Viruses (ICTV, paramyxovirus study group). Dr Lee has a special interest in emerging RNA viruses and HIV with a focus on molecular viral-host interactions that govern virus entry and budding.
\end{abstract}

First draft submitted: 8 April 2019; Published online: 17 July 2019

Keywords: diversity $\bullet$ ebola $\bullet$ ephrin-B $\bullet$ henipavirus $\bullet$ pandemic potential $\bullet$ paramxyovirus $\bullet$ pathogenicity $\bullet$ vaccine development

In this exclusive interview, Benhur Lee discusses his research into henipaviruses, highlighting the current knowns and unknowns. He considers how newly discovered divergent viral clades could help us to understand and uncover henipavirus pathogenicity determinants and the importance of this for future therapeutic development, in light of pandemic potentials. This interview was conducted by Ellen Colvin, Editor of Future Virology.

\section{Your research is focused on the diversity of Henipavirus \& its pathogenicity. Can you tell me a bit about this?}

Initially, when I began research, Hendra and Nipah viruses were the only two viruses that made up the Henipavirus genus. These were both pathogenic, with their own unique properties, and were classified under their own genus within the family of paramxyoviruses. The most commonly known paramxyoviruses that infect humans are measles and mumps viruses, which do cause disease but are rarely fatal. In contrast, henipaviruses, initially thought to be concentrated in South East Asia (SEA), cause fatal encephalitis in 40-90\% of infected individuals. Nipah and Hendra are distinct viruses but are closely related enough whereby if you make antibodies against Nipah virus, often, this will cross-react and cross-neutralize against Hendra virus and vice versa. My work tries to understand why these viruses are so virulent, how they spread, and how they cause such severe disease using relevant animal models. Being Biosafety Level 4 pathogens, there are obvious limits to the type and number of experiments that can be carried out. In the early days, henipaviruses were thought to be confined to SEA, so I was often questioned as to why I was working on a virus that is not present in this hemisphere (and may not even pose a health risk here). However, viral ecology groups on wildlife surveying missions discovered in 2012 that bats - the usual reservoir for Nipah and Hendra in SEA - remarkably and surprisingly harbored a whole host of paramyxoviruses, including many divergent clades of henipaviruses.

\section{How does diversity affect Henipavirus pathogenicity?}

Since 2012, at least 19 divergent clades of henipaviruses have been discovered in various viral surveys carried out by many groups. This revealed an entire universe of henipaviruses that were completely unknown, presenting

Future Medicine 
opportunities for us to try to determine whether any spillover events from bats to humans, that were previously unknown, had occurred. From this, we have serological evidence that some humans, such as bat butchers in Cameroon, have already been exposed to related henipaviruses and have consequently generated antibodies that cross-neutralize Nipah and Hendra virus. Although these African henipaviruses are distantly related to Asiatic Nipah and Hendra viruses, they appear to use the same major receptor, ephrin-B2. The more information that is obtained, the more we need to realize and understand that monitoring and surveillance is extremely important. Like members of the Ebolavirus genus, when more species are discovered, some will have pathogenic potential, but some won't. Understanding which of these can or cannot cause disease, will help give a better insight into the pathogenicity determinants and assist later in therapeutic and vaccine development. In the case of henipaviruses, the prototypical Nipah and Hendra viruses use ephrin-B2 and ephrin-B3 as receptors; ephrin-B3 is related to ephrin-B2 but is expressed more exclusively and at higher levels in the brain than ephrin-B2. We believe that ephrin-B3 usage plays a key role in the ability of Nipah and Hendra viruses to cause encephalitis. However, the one African henipavirus (Ghana virus) examined thus far, appears to use only ephrin-B2 but not ephrin-B3, while another divergent Australian henipavirus (Cedar virus) uses ephrin-B2 and ephrin-B1, but not ephrin-B3. All three ephrin-B members have distinctand overlapping tissue expression profiles. Thus, it is likely that the peculiarities of receptor usage will contribute to the spectrum of virulence amongst henipaviruses.

\section{Is there potential for Nipah \& Hendra pandemics in humans? What factors contribute to this?}

It has been clearly documented in Bangladesh and more recently, in Kerala (India), that Nipah virus is able to transmit directly from bats to humans, and from humans to humans, but there are still many unknowns. In the last outbreak in Kerala (India), there was a lot of press cover because such an outbreak in a big, densely populated city was cause for panic. How then, with the possibility of one person transmitting to ten others in a hospital, did it stop?

By gathering all the evidence, as it stands, Nipah and Hendra viruses are believed to transmit inefficiently. This may be either be a consequence of poor transmission by small aerosols or due to a possible need for extended contact with bodily fluids containing high viral loads for transmission. Whether or not the virus can mutate and adapt as it gets more opportunities to spread across and within human populations, is what is causing concern. CEPI, the coalition for epidemic preparedness innovations, is an organization that started after the Ebola outbreak in West Africa (2015). Key players in global health decided the response from the WHO was too slow and thus, CEPI, now with close to a US $\$ 1$ billion endowment fund from the Gates foundation, Wellcome Trust and other state players (such as Norway and India) wanted this to change. If you wait for a pharmaceutical firm to develop a vaccine for neglected diseases, it will never get done as too often, the risk is too high. CEPI's aim is to stockpile vaccines in preparation for an outbreak for these diseases.

When the Ebola epidemic happened, potential vaccines were already available that had been tested in nonhuman primates, but there was a lack of urgency to test them in human safety trials, with no incentive to make and stockpile effective vaccines for such 'neglected diseases'. Prior to the outbreak, there was no political will to test them and bring them to trial. After the outbreak occurred, suddenly vaccines came seemingly out of the blue and were being tested. CEPI wants to change this approach. They used the WHO's published 8-10 diseases of pandemic concern, in other words, viruses most likely to cause a pandemic, on which Nipah virus has been listed for years. CEPI's first call for vaccines was for Lassa fever virus, MERS coronavirus and Nipah virus. I was asked by the CEPI admin staff to give my opinions about Nipah virus, whereby I gave my recommendations and advice on a list of potential vaccines already published in the literature. I told them, controversially, if they were sure the next outbreak was to be Nipah or Hendra, then I believe we might already have effective vaccines, which work in animals, and I have no doubt that they will work in humans. There is a vaccine now for horses against Hendra virus because unlike a virus such as HIV, it is relatively easy to make a vaccine toward Nipah and Hendra viruses. However, myself and others have now shown that antibodies elicited against Nipah or Hendra viruses do not cross-neutralize (or cross-react) with many of the divergent Henipavirus clades recently discovered. Henipaviruses now form such divergent clades world-wide, including putative ones found in Central and South America, that any antibodies generated against Nipah and Hendra virus itself, will likely not protect against the divergent henipaviruses that we already know about. If any of these divergent strains jumps species barriers (or new ones that we don't know about), then there might be a problem. 
More research is required, and more effort needs to be put into generating (vaccines that will elicit) broadly neutralizing antibodies that will neutralize across the divergent clades of henipaviruses, which is what we are trying to do.

\section{What are your predictions for this field in the next 10-15 years? What do you hope to see?}

For Nipah and Hendra, we are clearly just beginning to touch the tip of the iceberg because there are many clades that exist in Africa, the Americas and even parts of East Asia (China). At present, we only have many snippets of different Henipavirus genes but it is not trivial to get the whole genome; more effort needs to be devoted to characterizing the entire genomes from these divergent clades because without this, it is impossible to determine what the virus does and how it works. One of the scarier or exciting things, depending on your view, is the related henipaviruses also being found in the new world, in Brazil and Costa Rica, and in insectivorous bats, with completely distinct ecology to Southeast Asian fruit bats. Thus, it is not just basic scientific research that is required, we also need viral and field ecologists to set up systems and join forces in order to try to better understand these things and to get a better handle on Henipavirus diversity, and the determinants of pathogenicity.

It is hard to make predictions, but for henipaviruses, based on what we know so far, I believe the use of ephrin-B2 has a major role in its systemic spread and multi-organ pathology. Ephrin-B2 is often found in small blood vessels that supply the brain and many other organs. I believe exploitation of this protein, and possibly of other ephrin-B ligands such as ephrin-B3, plays an important role in how it spreads, and how the virus causes inflammation in the brain (encephalitis). How well it can use a specific ephrin-B receptor and how receptor usage leads to the observed patient pathology is still something we need to work out.

\section{Financial \& competing interests disclosure}

The work discussed above is funded in part by NIH grants Al13449, Al1255362, Al115226, Al07647. The author has no other relevant affiliations or financial involvement with any organization or entity with a financial interest in or financial conflict with the subject matter or materials discussed in the manuscript apart from those disclosed.

No writing assistance was utilized in the production of this manuscript.

\section{Disclaimer}

The opinions expressed in this interview are those of B Lee and do not necessarily reflect the views of Future Medicine Ltd/Future Science Ltd/Newlands Press Ltd. 
